\title{
Post-Cesarean Delivery Analgesic Outcomes in Patients Maintained on Methadone and Buprenorphine: A Retrospective Investigation
}

This article was published in the following Dove Press journal: Journal of Pain Research

\author{
Joseph L Reno $\mathbb{D}^{\prime}$ \\ Michael Kushelev $\mathbb{D}^{2}$ \\ Julie H Coffman ${ }^{3}$ \\ Mona R Prasad ${ }^{4}$ \\ Avery M Meyer ${ }^{5}$ \\ Kristen M Carpenter ${ }^{5}$ \\ Marilly S Palettas ${ }^{6}$ \\ John C Coffman $\mathbb{D}^{2}$
}

'Department of Anesthesiology and Pain Medicine, University of Washington, Seattle, WA, USA; ${ }^{2}$ Department of Anesthesiology, The Ohio State University Medical Center, Columbus, $\mathrm{OH}$, USA; ${ }^{3}$ Department of Internal Medicine, Riverside Methodist Hospital, Columbus, OH, USA; ${ }^{4}$ Department of OBGYN, OhioHealth, Columbus, $\mathrm{OH}$, USA; ${ }^{5}$ Department of Psychiatry and Behavioral Health, The Ohio State University Wexner Medical Center, Columbus, OH, USA; ${ }^{6}$ Center for Biostatistics, The Ohio State University, Columbus, $\mathrm{OH}$, USA
Correspondence: Joseph L Reno

Tel +l 2065432673

Fax +l 2065432958

Email renoj@uw.edu
Background: Despite the increasing prevalence of opioid use disorder (OUD) in pregnant women, there are limited studies on their anesthesia care and analgesic outcomes after cesarean delivery (CD).

Methods: Patients with OUD on either buprenorphine or methadone maintenance therapy who underwent $C D$ at our institution from 2011 to 2018 were identified. Anesthetic details and analgesic outcomes, including daily opioid consumption and pain scores, were compared between patients maintained on buprenorphine and methadone. Analgesic outcomes were also evaluated according to anesthetic type (neuraxial or general anesthesia) and daily buprenorphine/methadone dose to determine if these factors impacted pain after delivery.

Results: A total of 146 patients were included (buprenorphine $n=99$ (67.8\%), methadone $\mathrm{n}=47(32.2 \%))$. Among all patients: $74 \%$ had spinal/CSE, $15 \%$ epidural, and $11 \%$ general anesthesia. Anesthesia types were similar among buprenorphine and methadone patients. For spinal anesthetics, intrathecal fentanyl (median $15 \mu \mathrm{g}$ ) and morphine (median $100 \mu \mathrm{g}$ ) were commonly given (97.2\% and $96.3 \%$, respectively), and dosed similarly between groups. Among epidural anesthetics, epidural morphine (median $2 \mathrm{mg}$ ) was commonly administered (90.9\%), while fentanyl (median $100 \mu \mathrm{g}$ ) was less common (54.5\%). Buprenorphine and methadone groups consumed similar amounts of oxycodone equivalents per 24 hours of hospitalization ( 80.6 vs $76.3 \mathrm{mg} ; p=0.694$ ) and had similar peak pain scores (8.3 vs 8.0; $p=0.518)$. Daily methadone dose correlated weakly with opioid consumption $(\mathrm{R}=0.3$; $p=0.03$ ), although buprenorphine dose did not correlate with opioid consumption or pain scores. General anesthesia correlated with greater oxycodone consumption in the first 24 hours (median $156.1 \mathrm{vs} 91.7 \mathrm{mg} ; p=0.004)$ and greater IV PCA use $(63 \%$ vs $7 \% ; p<0.001)$ compared to neuraxial anesthesia.

Conclusion: Patients on buprenorphine and methadone had similar high opioid consumption and pain scores after CD. The anesthetic details and analgesic outcomes reported in this investigation may serve as a useful reference for future prospective investigations and aid in the clinical care of these patients.

Keywords: opioid use disorder, methadone, buprenorphine, cesarean analgesia, outcomes, enhanced recovery

\section{Introduction}

Opioid use disorder (OUD) in the obstetric population has steadily increased in prevalence in the United States, with one report citing an increase of $127 \%$ between 1998 and 2011. ${ }^{1}$ Despite the increasing incidence of OUD in pregnant women, currently there is a paucity of evidence on how to provide adequately for their 
unique anesthetic and analgesic needs, especially after cesarean delivery. $^{2-5}$ Women with OUD are commonly excluded from prospective studies, and current knowledge rests on a few retrospective investigations of analgesic outcomes, but these reports lack anesthetic details relevant to pain after delivery. ${ }^{6-11}$ Existing literature on perioperative handling of buprenorphine or methadone does not specifically discuss obstetrics. ${ }^{12-14}$

Retrospective studies of postpartum analgesia in OUD populations usually relate analgesic regimens of oxycodone, non-steroidal anti-inflammatory drugs (NSAIDs), and acetaminophen with clinically practical, easily tracked analgesic outcomes such as verbal pain scores and daily opioid consumption (often standardized to oxycodone equivalents consumed). ${ }^{6-8,10}$ In existing obstetric cohorts, the delivery method is often noted, but anesthetic details are not. $^{4-10}$

This study focuses on several analgesic outcomes: 24hour opioid requirements, pain scores, time to first postoperative pain medication received, delivery-to-discharge interval, intravenous (IV) patient-controlled analgesia (PCA) use, and truncal block use. It reports on how these outcomes differ by use of buprenorphine versus methadone, anesthetic type (neuraxial versus general anesthesia), and by daily maintenance therapy dose. Our large cohorts reflect historic anesthetic practice, and inclusion of buprenorphine and methadone doses enables their appraisal as possible predictors for analgesia after cesarean delivery. Reporting anesthetic details along with analgesic outcomes establishes an important baseline understanding of the unique analgesic needs of OUD patients after cesarean delivery, and this understanding will likely be useful in developing future prospective studies and may aid clinicians tasked with caring for these patients.

\section{Methods}

This is a single-institution, retrospective study of women cared for in the Substance abuse Treatment Education and Prevention Program (STEPP) at The Ohio State University Wexner Medical Center from December 2011 to February 2018. STEPP clinic has provided maintenance treatment, prenatal care, education, and group counseling to pregnant and postpartum women with OUD since 2010. A list of obstetric patients was obtained from STEPP clinic records during the study period. Because of the retrospective nature of this study, no signed patients' consent forms were required by the Ohio State University Institutional Review Board (IRB). After IRB approval, patient data were abstracted from patients' electronic medical records by approved study personnel and all data were stored in a password-protected electronic research database created for this study. Patient confidentiality was maintained and this study was conducted in accordance with the Declaration of Helsinki. Subjects included were those aged 18-44 maintained on methadone or buprenorphine therapy at the time of delivery who underwent cesarean delivery at our institution. Patients not delivering at our institution were excluded. Patients were also excluded if they underwent cesarean-hysterectomy, as this is expected to complicate postpartum analgesia. The objective of this investigation was to examine the anesthetic details and postcesarean analgesic outcomes in women treated with buprenorphine versus methadone. The primary outcomes of interest were 24-hour opioid consumption (expressed in oral oxycodone equivalents $(\mathrm{mg})$ ) and maximum pain scores each day. ${ }^{15}$ Secondary objectives included summarizing analgesic outcomes between types of anesthesia (neuraxial versus general) and assessing the correlation between daily dose of buprenorphine or methadone and opioid consumption or peak pain score after cesarean delivery. Commonly utilized spinal medication dosing ranges for cesarean anesthesia at our institution during the study period were spinal bupivacaine $12-13.5 \mathrm{mg}$, fentanyl 10-15 $\mu \mathrm{g}$, and morphine 100-150 $\mu \mathrm{g}$. Commonly used epidural medication dosing ranges included: local anesthetic 15-20 mL (in $5 \mathrm{~mL}$ increments), fentanyl $50-100 \mu \mathrm{g}$, and morphine $2 \mathrm{mg}$. Since this was a retrospective investigation, adherence to a common anesthetic regimen was not required. Postpartum monitoring on the inpatient ward included vital signs including respiratory rate and sedation assessment every 4 hours.

Maternal and fetal characteristics included age, weight, gravidity, parity, number of previous term and preterm deliveries, previous cesarean deliveries, gestational age at index delivery, smoking status, marital status, race, hepatitis C or HIV comorbidity, daily buprenorphine or methadone dose at delivery (both were continued perioperatively), birth weight, and Apgar scores at 1 and 5 minutes (Table 1). These demographics allowed comparison of our cohorts to previous studies' cohorts. ${ }^{1,6-8}$

Anesthetic characteristics included type of anesthesia (spinal/combined spinal-epidural (CSE), epidural, or general), dose of spinal medications (bupivacaine, morphine, fentanyl, epinephrine, or clonidine), dose of epidural medications administered (volume of local anesthetic, morphine dose, fentanyl dose), reason for general anesthesia, need for 
Table I Demographic and Obstetric Characteristics by Buprenorphine/Methadone Group

\begin{tabular}{|c|c|c|c|c|}
\hline & & Buprenorphine $\mathrm{n}=99$ & Methadone $n=47$ & $P$-value \\
\hline \multicolumn{2}{|c|}{ Mother's age at delivery (years) } & $28[24,31]$ & $27[24,30]$ & 0.300 \\
\hline \multirow[t]{4}{*}{ Mother's race } & Black & $8(8 \%)$ & $2(4 \%)$ & 0.376 \\
\hline & Hispanic & I (I\%) & $2(4 \%)$ & \\
\hline & Non-Hispanic White & $89(90 \%)$ & $42(89 \%)$ & \\
\hline & Other & $\mathrm{I}(\mathrm{l} \%)$ & I (2\%) & \\
\hline \multicolumn{2}{|c|}{ Mother's weight (kg) } & $76.2[68.9,91.6]$ & $79.8[69.9,92.5]$ & 0.548 \\
\hline \multirow{2}{*}{\multicolumn{2}{|c|}{$\begin{array}{l}\text { Gravidity } \\
\text { Parity }\end{array}$}} & $4[2,5]$ & $3[2,4]$ & 0.015 \\
\hline & & $2[1,3]$ & $\mathrm{I}[\mathrm{I}, 2]$ & 0.044 \\
\hline \multicolumn{2}{|c|}{ Previous term deliveries } & $\mathrm{I}[\mathrm{I}, 2]$ & I $[0,2]$ & 0.105 \\
\hline \multicolumn{2}{|c|}{ Previous preterm deliveries } & $0[0,1]$ & $0[0,0]$ & 0.384 \\
\hline \multicolumn{2}{|c|}{ Previous cesarean deliveries } & I $[0,2]$ & $0[0,1]$ & 0.050 \\
\hline \multicolumn{2}{|c|}{ Buprenorphine/methadone daily dose at delivery (mg) } & $16[16,16]$ & $110[80,130]$ & - \\
\hline \multicolumn{2}{|l|}{ Medicaid } & 89 (90\%) & 43 (9l\%) & 0.950 \\
\hline \multirow[t]{2}{*}{ Marital status } & Divorced & $8(8 \%)$ & $4(9 \%)$ & 0.965 \\
\hline & Single & 77 (78\%) & $38(81 \%)$ & \\
\hline \multicolumn{2}{|l|}{ Smoking } & 85 (86\%) & 39 (83\%) & 0.491 \\
\hline \multicolumn{2}{|c|}{ Depression history } & $35(35 \%)$ & $16(34 \%)$ & 0.877 \\
\hline \multicolumn{2}{|c|}{ Hepatitis C } & $37(37 \%)$ & $23(49 \%)$ & 0.185 \\
\hline \multicolumn{2}{|l|}{ HIV } & I (I\%) & $0(0 \%)$ & 0.489 \\
\hline \multicolumn{2}{|c|}{ Planned cesarean delivery } & 68 (69\%) & $23(49 \%)$ & 0.021 \\
\hline \multicolumn{2}{|c|}{ Gestational age at delivery (weeks) } & $39[37,39]$ & $38[36,39]$ & 0.010 \\
\hline \multicolumn{2}{|l|}{ Birth weight (g) } & $2970[2565,3425]$ & $2820[2455,3420]$ & 0.349 \\
\hline \multicolumn{2}{|c|}{ Apgar I minute } & $8[8,9]$ & $8[7,9]$ & 0.010 \\
\hline \multicolumn{2}{|c|}{ Apgar 5 minutes } & $9[9,9]$ & $9[8,9]$ & 0.123 \\
\hline
\end{tabular}

Notes: Data shown are $\mathrm{n}(\%)$ for binary outcomes, and median [25th to 75 th percentile range] otherwise.

intraoperative supplemental IV analgesia or sedation, truncal block use, and anesthesia complications (Table 2). Patient charts were reviewed for the following anesthesia complications: inadvertent dural puncture, post-dural puncture headache, neurologic injury, failed intubation, aspiration, failed neuraxial block, or postoperative respiratory depression.

Analgesic outcomes included 24-hour opioid consumption in oxycodone equivalents (mg), highest and lowest pain scores each day, delivery-to-discharge interval (days), time to first analgesic received after surgery (minutes), incidence of IV PCA use, cumulative NSAID doses (ketorolac, ibuprofen), daily oral acetaminophen dose, and hospital readmission (Table 3). Recorded pain assessments did not permit calculation of time-weighted pain. Pain assessments were usually every 4 hours while awake and on the postpartum ward. Opioid consumption was reported as oxycodone equivalents (oral oxycodone $5 \mathrm{mg}=7.5 \mathrm{mg}$ oral morphine equivalents (OME) $)^{15}$ because this is the most commonly administered medication for breakthrough pain after cesarean delivery in many practices in the United States so is likely be clinically relevant to many providers. Also, oxycodone equivalents were reported in previous retrospective studies on analgesic consumption after cesarean delivery in OUD patients. ${ }^{6,7}$ At our institution, the common postpartum analgesic regimen included IV hydromorphone if needed in the recovery area, oral oxycodone as needed for breakthrough pain, scheduled IV ketorolac until 24 hours postpartum followed by scheduled oral ibuprofen from 24 hours postpartum until hospital discharge, and oral acetaminophen as needed. Initial oral oxycodone dosing range was typically 5-10 $\mathrm{mg}$ (at the discretion of the nurse depending on the pain level expressed by the patient), and these dosing ranges were escalated by physicians as needed for improved pain relief. IV PCA was reserved for patients with severe refractory pain. 
Table 2 Anesthetic Details by Buprenorphine/Methadone Group

\begin{tabular}{|c|c|c|c|}
\hline & Buprenorphine $\mathrm{n}=99$ & Methadone $n=47$ & $P$-value \\
\hline Spinal or CSE, $n=108$ (74\% of anesthetics) & $73(73.7 \%)$ & 35 (74.5\%) & 0.995 \\
\hline IT bupivacaine $(\mathrm{mg}), \mathrm{n}=108(100 \%)$ & $12[12,13.5]$ & $12[12,12]$ & 0.016 \\
\hline IT fentanyl $(\mu \mathrm{g}), \mathrm{n}=105$ (97.2\%) & $15[15,15]$ & $15[15,15]$ & 0.650 \\
\hline IT morphine $(\mu g), n=104(96.3 \%)$ & $100[100,150]$ & $100[100,100]$ & 0.498 \\
\hline IT epinephrine $(\mu \mathrm{g}), \mathrm{n}=10$ (9.3\%) & $75[50,200]$ & $100[50,100]$ & 0.890 \\
\hline IT clonidine $(\mu \mathrm{g}), \mathrm{n}=2(\mathrm{I} .9 \%)$ & $50[50,50]$ & - & - \\
\hline CSE epidural dose $(\mathrm{mL}), \mathrm{n}=\mathrm{I} \mid(\mathrm{I} 0.2 \%)$ & $5[0,10]$ & $3[3,20]$ & 0.679 \\
\hline Epidural, n=22 ( $15 \%)$ & $15(15.2 \%)$ & 7 (14.9\%) & 0.995 \\
\hline Epidural local anesthetic dose $(\mathrm{mL})$ & $17[15,20]$ & $15[15,20]$ & 0.435 \\
\hline Epidural fentanyl $(\mu \mathrm{g}), \mathrm{n}=12$ (54.5\%) & $100[90,100]$ & $100[85,100]$ & 0.929 \\
\hline Epidural morphine $(\mathrm{mg}), \mathrm{n}=20$ (90.9\%) & $2[2,2]$ & $2[2,2]$ & 0.690 \\
\hline $\begin{array}{l}\text { General anesthesia, } n=16 \text { ( I l\%) } \\
\text { Emergency, } n=6(37.5 \%) \\
\text { Neuraxial failed, } n=4(25 \%) \\
\text { Neuraxial contraindicated, } n=6 \text { (37.5\%) }\end{array}$ & II (II.I\%) & $5(10.6 \%)$ & 0.995 \\
\hline IV fentanyl or hydromorphone used intraoperatively & $26(26.3 \%)$ & $14(30.4 \%)$ & 0.600 \\
\hline IV hydromorphone used & $9(9.1 \%)$ & $5(10.9 \%)$ & 0.740 \\
\hline Hydromorphone dose (mg) & $2[2,2]$ & $2[2,2]$ & 0.880 \\
\hline IV anxiolytic/non-opioid analgesic used intraoperatively & $53(53.4 \%)$ & $24(52.2 \%)$ & 0.880 \\
\hline Nerve block used, n (\%) & $4(4 \%)$ & I (2\%) & 0.553 \\
\hline Anesthesia complications $^{\mathrm{a}}$ & $4(4 \%)$ & $2(4.3 \%)$ & 0.950 \\
\hline Inadequate spinal block & 4 & 1 & \\
\hline Inadequate epidural replaced & & I & \\
\hline
\end{tabular}

Notes: Data shown are $\mathrm{n}(\%)$ for binary outcomes, and median [25th to 75 th percentile range] otherwise. ${ }^{\mathrm{a}}$ Complications: inadvertent dural puncture, post-dural puncture headache, neurologic injury, failed intubation, aspiration, failed neuraxial block, postoperative respiratory depression, or naloxone administration.

Patient data were also analyzed by anesthesia type (neuraxial versus general), and the same analgesic outcomes were examined (Table 4). In order to examine possible correlation between daily maintenance therapy dose and analgesic outcomes, daily buprenorphine or methadone doses were plotted against oxycodone equivalents consumed per 24 hours of hospitalization (Figure 1), median daily maximum pain scores (Figure 2), and oxycodone consumption from 0-24 hours after delivery (Figure 3).

\section{Statistical Methods}

Patient characteristics as well as anesthetic details and analgesic outcomes were summarized using descriptive statistics. Comparisons between buprenorphine and methadone patients were made using a test of proportions or Wilcoxon rank-sum test, both two-sided, for binary and continuous outcomes, respectively. The same tests were used to summarize and compare outcomes by anesthetic types. Pain scores were reported as medians in keeping with the discrete, non-continuous nature of the $0-10$ verbal pain scale. Pearson's correlation coefficients and scatter plots were used to evaluate the relationship between buprenorphine or methadone dose against opioid consumption and pain scores. To control for additional factors, linear regressions were also used. Additional factors include all variables in Table 1 (other than Medicaid status for which there are seven missing data points; Apgar scores are included although data are missing for one patient). These variables were used as controls in the regressions, or to construct a propensity score which was subsequently included. From the linear regressions, we compute minimum detectable effects assuming a power of $80 \%$ and a significance threshold of 0.05 . Analyses were performed in SAS 9.4 (SAS Institute, Cary, NC, USA), in $\mathrm{R}$ version 3.5.2 (The $\mathrm{R}$ Foundation for Statistical Computing, Vienna, Austria), and in StataMP 16 (Stata Corp., College Station, TX, USA).

\section{Results}

A total of 146 women met the inclusion criteria for analysis. One patient was excluded for cesarean-hysterectomy due to intraoperative bleeding. Of the included patients, 
Table 3 Analgesic Outcomes by Buprenorphine/Methadone Group

\begin{tabular}{|c|c|c|c|}
\hline & Buprenorphine $n=99$ & Methadone $n=47$ & $P$-value \\
\hline Delivery-to-discharge interval (days) & $3[3,4]$ & $3[3,4]$ & 0.555 \\
\hline Time to first postoperative opioid request (minutes) & $64[34,106]$ & $79[49,138]$ & 0.140 \\
\hline Oxycodone equivalents per 24 hours of hospitalization & $80.6[62,108.3]$ (range & $76.3[63,105]$ (range & 0.694 \\
\hline (mg) & 7.9-257.5) & $5-167.3)$ & \\
\hline Postoperative day 0 (0-24 hours) & $100[75,126.7]$ & $83.3[63,158.3]$ & 0.358 \\
\hline Postoperative day I (24-48 hours) & $80[60,115]$ & $75[50,90]$ & 0.185 \\
\hline Postoperative day 2 (48-72 hours) & $70[40,100]$ & $75[50,92.7]$ & 0.988 \\
\hline Postoperative day 3 (72-96 hours) & $60[30,90]$ & $55[43,85]$ & 0.736 \\
\hline Highest pain score each day (verbal 0-10 scale) & $8.3[7,9]$ (range $5-10)$ & $8.0[7,8.7]$ (range $5.3-10)$ & 0.518 \\
\hline Postoperative day 0 (0-24 hours) & $9[8,10]$ & $9[8,10]$ & 0.816 \\
\hline Postoperative day I (24-48 hours) & $8[7,9]$ & $8[7,9]$ & 0.631 \\
\hline Postoperative day 2 (48-72 hours) & $8[6,9]$ & $8[6,8.5]$ & 0.699 \\
\hline Postoperative day 3 (72-96 hours) & $7[6,8]$ & $8[6,9]$ & 0.247 \\
\hline Lowest pain score each day (verbal 0-10 scale) & $2.5[1.5,3.5]$ & $2.1[1.3,3.3]$ & 0.510 \\
\hline Postoperative day 0 ( $0-24$ hours) & $3[2,5]$ & $3[1.5,4]$ & 0.280 \\
\hline Postoperative day I (24-48 hours) & $2[0,4]$ & $2.5[0.5,4]$ & 0.740 \\
\hline Postoperative day 2 ( $48-72$ hours) & $3[0,4]$ & $2[0,4]$ & 0.900 \\
\hline Postoperative day 3 (72-96 hours) & $0[0,3]$ & $0[0,4]$ & 0.950 \\
\hline Ketorolac cumulative dose $(\mathrm{mg})$ & $120[120,120]$ & $120[120,120]$ & 0.799 \\
\hline Ibuprofen cumulative dose (mg) & $3200[2667,3200]$ & $3200[2800,3200]$ & 0.676 \\
\hline Acetaminophen daily dose (mg) & $1083[433,1950]$ & $650[379,1110]$ & 0.022 \\
\hline IV PCA use, n (\%) & $8(8 \%)$ & II (23\%) & 0.010 \\
\hline Hospital readmission, n (\%) & $2(2 \%)$ & I (2\%) & 0.970 \\
\hline Infection & I & I & \\
\hline Bowel obstruction & I & & \\
\hline
\end{tabular}

Notes: Data shown are $\mathrm{n}(\%)$ for binary outcomes, and median [25th to 75th percentile range] otherwise.

$67.8 \%$ were maintained on buprenorphine $(\mathrm{n}=99$; median daily dose $16 \mathrm{mg}$ [interquartile range (IQR) 16, 16]) and $32.2 \%$ were maintained on methadone $(n=47$; median daily dose $110 \mathrm{mg}[80,130])$ at the time of cesarean delivery. The number of patients maintained on buprenorphine and methadone undergoing cesarean delivery each year of the study period is shown in Figure 4. The buprenorphine and methadone groups in this retrospective study differed in several ways: gravidity, parity, number of prior cesareans, and incidence of planned cesarean deliveries were greater in the buprenorphine group. The buprenorphine group delivered at greater gestational age (median $39[37,39]$ vs $38[36,39]$ weeks; $p=0.010$ ) and had higher 1-minute Apgar scores (median $8[8,9]$ vs 8 $[7,9] ; p=0.010$ ) (Table 1).

Anesthetic management was similar between buprenorphine vs methadone groups (Table 2), with similar rates of spinal/CSE $(73.7 \%$ vs $74.5 \% ; p=0.995)$, epidural $(15.2 \%$ vs $14.9 ; p=0.995)$, and general anesthesia $(11.1 \%$ vs
$10.6 \% ; p=0.995)$. The buprenorphine group had slightly higher spinal bupivacaine doses ((median $12[12,13.5]$ vs $12[12,12] ; p=0.016)$, but anesthetic medication doses were otherwise statistically similar. Among all patients receiving spinal or CSE anesthesia, most received spinal bupivacaine $(100 \%)$, fentanyl $(97.2 \%)$, and morphine (96.3\%). Spinal epinephrine (9.3\%) and clonidine (1.9\%) were not commonly administered. Among all patients receiving epidural anesthesia ( $\mathrm{n}=22,15 \%$ of anesthetics), epidural morphine was commonly given $(90.9 \%)$ while only about half received epidural fentanyl (54.5\%). General anesthesia was performed in $11 \%$ of patients $(n=16)$ for the following indications: emergency/inadequate time for neuraxial block $(n=6)$, failed neuraxial block $(n=4)$, and contraindication to neuraxial $(n=6)$. Truncal nerve blocks, which included transversus abdominis (TAP) and ilioinguinal-iliohypogastric (II-IH), were performed in $4 \%$ and $2 \%$ of the buprenorphine and methadone groups, respectively $(p=0.553)$. 
Table 4 Analgesic Outcomes by Anesthetic Type

\begin{tabular}{|c|c|c|c|}
\hline & Neuraxial $n=130$ & General $n=16$ & $P$-value \\
\hline IV fentanyl or hydromorphone used intraoperatively & 25 (19.4\%) & $15(93.8 \%)$ & $<0.001$ \\
\hline IV hydromorphone used & $2(1.6 \%)$ & $12(75 \%)$ & $<0.001$ \\
\hline Hydromorphone dose (mg) & $2[2,2]$ & $2[1.8,2]$ & 0.830 \\
\hline IV anxiolytic/non-opioid analgesic used intraoperatively & $66(51.2 \%)$ & II (68.8\%) & 0.180 \\
\hline Delivery-to-discharge interval (days) & $3[3,4]$ & $3[3,4]$ & 0.510 \\
\hline Time to first postoperative opioid request (minutes) & $67[46,114]$ & $58[30.5,113.5]$ & 0.340 \\
\hline Oxycodone equivalents per 24 hours of hospitalization & $79.6[63,105]$ (range & $87.6[70,134.3]$ (range & 0.222 \\
\hline$(\mathrm{mg})$ & $5-257.5)$ & $31.3-221.7)$ & \\
\hline Postoperative day 0 (0-24 hours) & $91.7[70,121.7]$ & $156.1[104,221.7]$ & 0.004 \\
\hline Postoperative day I (24-48 hours) & $80[60,110]$ & $62.5[48,92.5]$ & 0.210 \\
\hline Postoperative day 2 (48-72 hours) & $75[45,100]$ & $75[40,90]$ & 0.685 \\
\hline Postoperative day 3 (72-96 hours) & $60[30,90]$ & $50[43,115]$ & 0.620 \\
\hline Highest pain score each day (verbal $0-10$ scale) & $8.3[7,9]$ (range 5-10) & $7.8[7,9]$ (range 6-10) & 0.931 \\
\hline Postoperative day 0 (0-24 hours) & $9[8,10]$ & $10[10,10]$ & 0.114 \\
\hline Postoperative day I (24-48 hours) & $8[7,9]$ & $8[7,10]$ & 0.574 \\
\hline Postoperative day 2 (48-72 hours) & $8[6,9]$ & $7[6,8]$ & 0.332 \\
\hline Postoperative day 3 (72-96 hours) & $7[6,8]$ & $7.5[6,8]$ & 0.845 \\
\hline Lowest pain score each day (verbal $0-10$ scale) & $2.3[1.3,3.5]$ & $2.4[1.5,3]$ & 0.840 \\
\hline Postoperative day 0 (0-24 hours) & $3[2,4]$ & $4[2.5,6]$ & 0.270 \\
\hline Postoperative day I (24-48 hours) & $2[0,4]$ & $3[0,3]$ & 1.00 \\
\hline Postoperative day 2 (48-72 hours) & $2.5[0,4]$ & $2[0,3]$ & 0.360 \\
\hline Postoperative day 3 (72-96 hours) & $0[0,4]$ & $0[0,0]$ & 0.290 \\
\hline Ketorolac cumulative dose (mg) & $120[120,120]$ & $120[90,120]$ & $0.44 I$ \\
\hline Ibuprofen cumulative dose (mg) & $3200[2667,3200]$ & $3200[2667,3200]$ & 0.839 \\
\hline Acetaminophen daily dose (mg) & $975[433,1733]$ & $1002[542,1463]$ & 0.940 \\
\hline IV PCA used, n (\%) & $9(7 \%)$ & $10(63 \%)$ & $<0.001$ \\
\hline Nerve block used, n (\%) & $5(4 \%)$ & $0(0 \%)$ & 1.000 \\
\hline
\end{tabular}

Notes: Data shown are $\mathrm{n}(\%)$, and median [25th to 75th percentile range] otherwise.

Buprenorphine and methadone groups had similar analgesic outcomes, including: 24-hour opioid consumption, maximum and minimum pain scores each day, time to first postoperative opioid medication received, and delivery-to-discharge intervals (Table 3). Buprenorphine patients used more acetaminophen (median daily dose (mg) $1083[433,1950]$ vs $650[379,1110] ; p=0.022)$ and had lower IV PCA use ( $8 \%$ vs $23 \%$; $p=0.010)$. Notably, both buprenorphine and methadone groups had similar high peak pain scores (median 8.3 [7, 9] vs 8.0 [7, 8.7]; $p=0.518$ ). Oral oxycodone equivalent $(\mathrm{mg})$ consumption per 24 hours of hospitalization was also similar between groups (median 80.6 [62, 108.3] vs 76.3 [63, 105]; $p=0.694$ ). No patients required readmission for pain control.

The daily buprenorphine and methadone doses for each patient were plotted against oxycodone equivalent consumption per 24 hours of hospitalization (Figure 1A and
B), maximum pain scores each day (Figure 2A and B), and opioid consumption in the first 24 hours postoperatively (Figure 3A and B). Daily methadone dose showed weak correlation to oxycodone equivalent consumption per 24 hours after delivery $(\mathrm{R}=0.32 ; p=0.03)$. Otherwise, methadone dose showed no significant correlation with pain scores or first 24 hours postoperative opioid requirement. Buprenorphine dose showed no correlation with any of these variables.

Analgesic outcomes were also compared between patients receiving neuraxial (spinal, CSE, or epidural) versus general anesthesia (Table 4). Neuraxial anesthesia patients had lower oral oxycodone equivalent $(\mathrm{mg})$ consumption in the first 24 hours $(91.7$ [70, 121.7] mg vs 156.1 [104, 221.7]; $p=0.004)$ and less IV PCA use $(7 \%$ vs $63 \% ; p<0.001)$ compared to patients receiving general anesthesia. Supplemental IV opioids (fentanyl or hydromorphone) were administered more frequently to patients receiving general compared to 
A

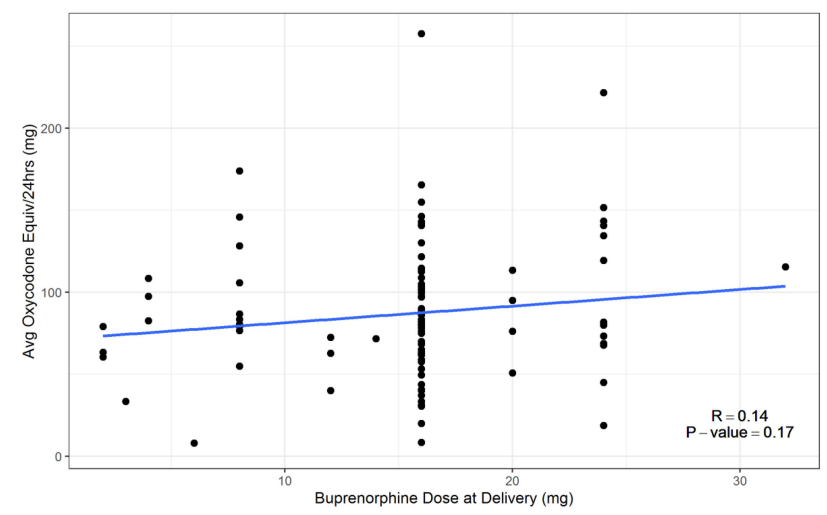

B

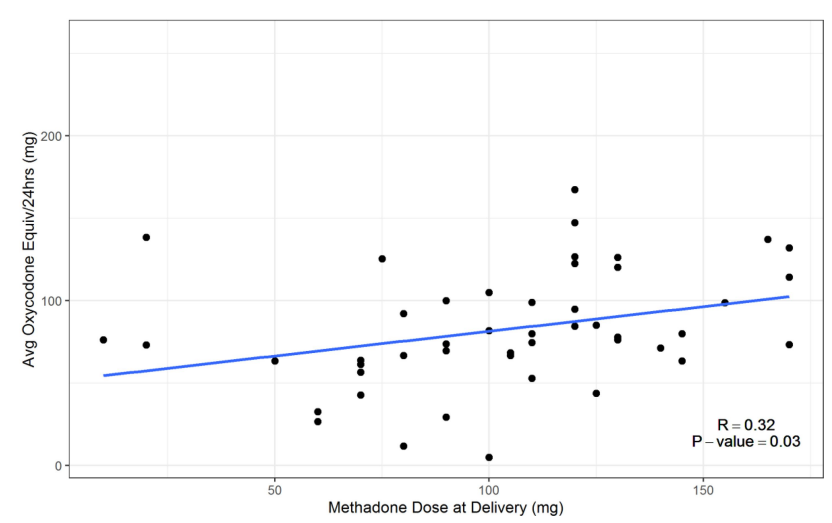

Figure I Maintenance agent dose at delivery compared to oxycodone equivalents per 24 hours of hospitalization after cesarean delivery. (A) Buprenorphine dose (mg) compared to oxycodone equivalents per 24 hours of hospitalization $(\mathrm{mg})$. (B) Methadone dose $(\mathrm{mg})$ compared to oxycodone equivalents per 24 hours of hospitalization (mg).

Abbreviations: $\mathrm{R}$, correlation; $P$-value, statistical significance.

A

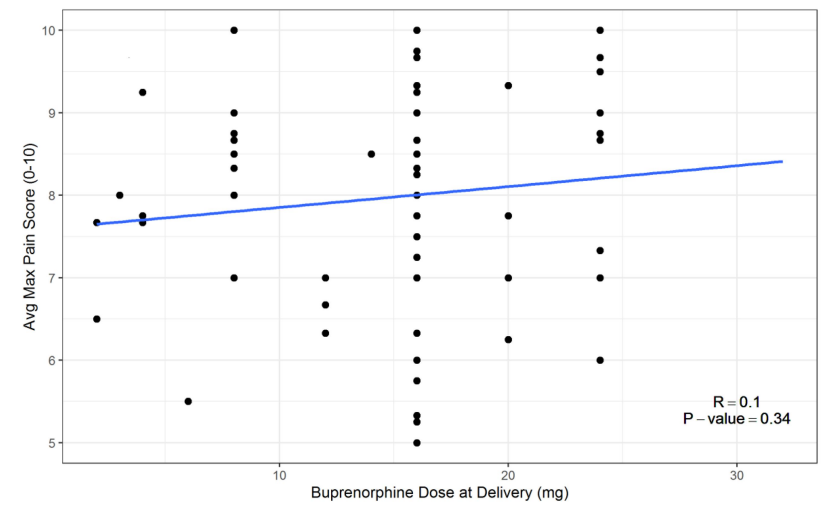

B

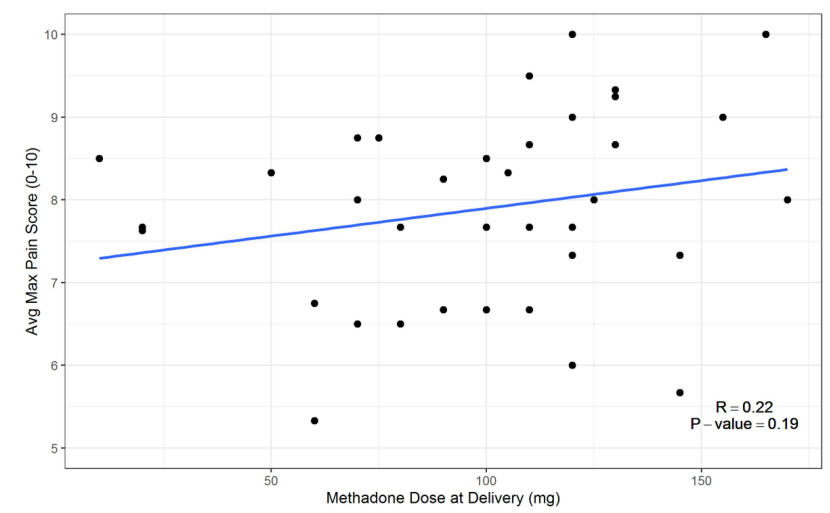

Figure 2 Maintenance agent dose at delivery compared to peak pain score after cesarean delivery. (A) Buprenorphine dose in milligrams (mg) compared to the average of all days' highest verbal pain score (rated 0-10). (B) Methadone dose $(\mathrm{mg})$ compared to the average of all days' highest verbal pain score (rated 0-10).

Abbreviations: R, correlation; $P$-value, statistical significance.

neuraxial anesthesia (93.8\% vs $19.4 \% ; p<0.001)$. IV hydromorphone, a longer acting IV opioid, was also given more frequently intraoperatively for those getting general anesthesia compared to neuraxial anesthesia ( $75 \%$ vs $1.6 \%$; $p<0.001)$. Notably, over $50 \%$ of patients required supplemental intraoperative anxiolysis in all groups. No other differences were detected in pain scores, opioid consumption, or multimodal analgesic measures.

The null effect between buprenorphine and methadone patients on analgesic outcomes does not change when additional factors are considered (Table 5). The effect of administering methadone instead of buprenorphine on 24hour oxycodone $(\mathrm{mg})$ during hospitalization, oxycodone (mg) in the first 24 postoperative hours, highest daily pain score, and lowest daily pain score is statistically indistinguishable from zero for all specifications considered. Additionally, the minimum detectable effect in all regressions is between one-fourth and one-fifth of a standard deviation in the outcome suggesting sufficient statistical power to identify a meaningful effect had one existed. Likewise, the regressions with controls find no effects on our outcomes of interest comparing general anesthesia instead of neuraxial anesthesia, other than, as above, oxycodone administered in the first 24 hours (Table 5).

\section{Discussion}

This 8- year retrospective cohort study observed several demographic similarities to previous studies in obstetric patients with OUD, as we observed patients to be 


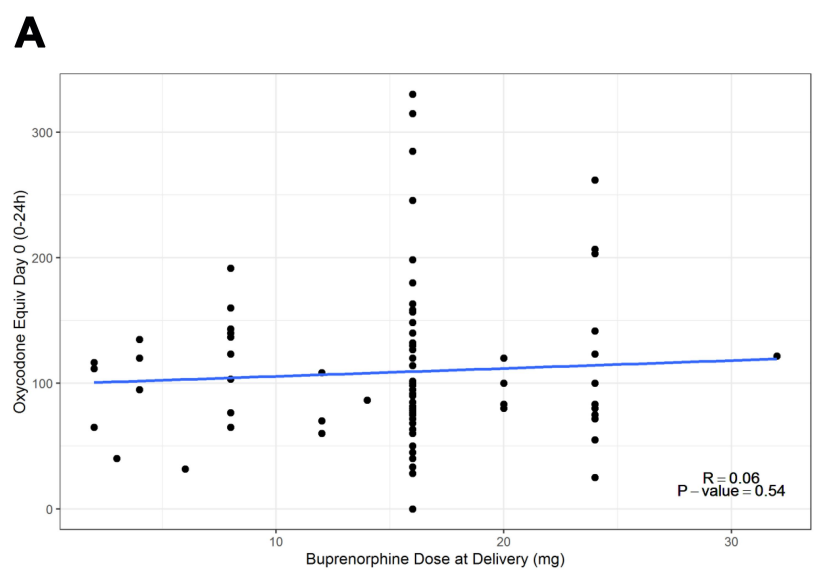

B

Figure 3 Maintenance agent dose at delivery compared to oxycodone equivalents taken from 0-24 hours after cesarean delivery. (A) Buprenorphine dose in milligrams ( $\mathrm{mg}$ ) compared to oxycodone equivalents $(\mathrm{mg})$ during first 24 hours post cesarean delivery. (B) Methadone dose $(\mathrm{mg})$ compared to oxycodone equivalents (mg) during first 24 hours post cesarean delivery.

Abbreviations: $\mathrm{R}$, correlation; $P$-value, statistical significance.
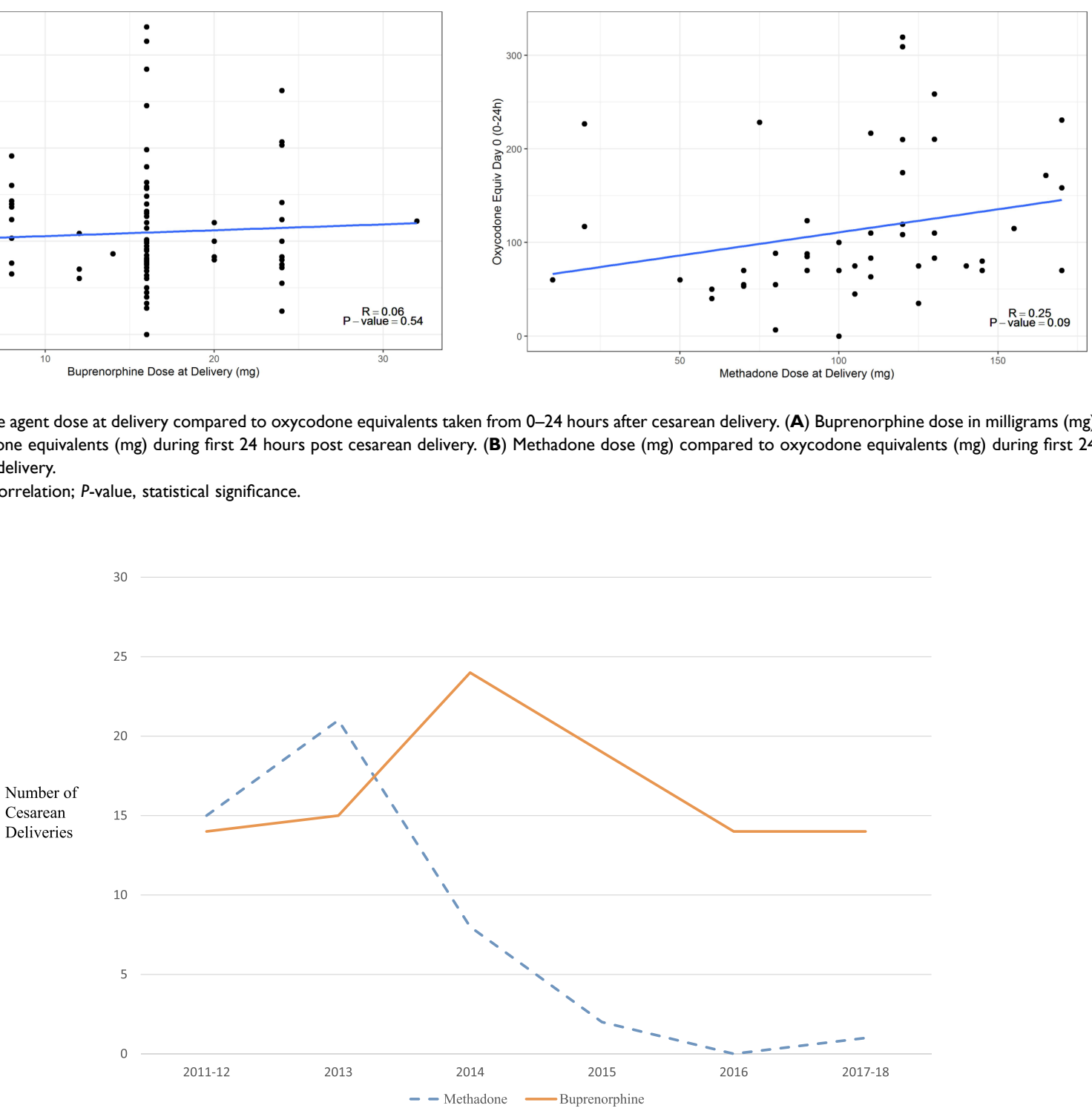

Figure 4 Number of cesarean deliveries per year in patients maintained on buprenorphine (solid line) and methadone (dotted line). Note that the study period was December $201 \mathrm{I}$ until February 2018. For the purposes of this figure 20II-12 are grouped together and 2017-18 are grouped together, since only one month of 20II and two months of 2018 are included in this study.

predominantly white, unmarried, on Medicaid insurance, and have a relatively high incidence of smoking, depression, and hepatitis C. ${ }^{1,6-8}$ Several key differences in the buprenorphine group complicate our results: gravidity, parity, previous cesarean deliveries, and gestational age at delivery were higher. This group also had a greater chance of undergoing planned cesarean, although one study found this factor not to affect analgesia. ${ }^{16}$ These asymmetries generally suggest a potentially more painful postpartum course for the buprenorphine group, although the buprenorphine group also received more acetaminophen. Notwithstanding these differences, we found pain scores and opioid consumption did not differ between patients taking buprenorphine vs methadone, similar to previous reports. ${ }^{8}$ Further, controlling for the differences discussed above, we find no differences in the buprenorphine vs methadone groups on pain scores or opioid consumption (Table 5). The notably high 24-hour oxycodone consumption in both buprenorphine and methadone groups (median 80.6 vs $76.3 \mathrm{mg}$ per day, respectively; $p=0.694$, see Table 3 ) shows that neither group achieved satisfactory analgesia. For reference, oral 
Table 5 Ordinary Least Squares Regressions of Analgesic Outcomes

\begin{tabular}{|c|c|c|c|c|c|c|c|c|}
\hline \multirow[b]{2}{*}{ Methadone } & \multicolumn{2}{|c|}{$\begin{array}{l}\text { Oxycodone Equivalents } \\
(\mathrm{mg}) \text { per } 24 \text { hours of } \\
\text { Hospitalization }\end{array}$} & \multicolumn{2}{|c|}{$\begin{array}{l}\text { Oxycodone Dose }(\mathrm{mg}) \\
0-24 \text { hours }\end{array}$} & \multicolumn{2}{|c|}{$\begin{array}{l}\text { Highest Daily Pain } \\
\text { Score }(0-10)\end{array}$} & \multicolumn{2}{|c|}{$\begin{array}{l}\text { Lowest Daily Pain } \\
\text { Score }(0-10)\end{array}$} \\
\hline & $\begin{array}{l}1.26 \\
(6.08)\end{array}$ & $\begin{array}{l}1.62 \\
(7.48)\end{array}$ & $\begin{array}{l}9.03 \\
(9.81)\end{array}$ & $\begin{array}{l}11.76 \\
(12.13)\end{array}$ & $\begin{array}{l}0.05 \\
(0.24)\end{array}$ & $\begin{array}{l}0.07 \\
(0.30)\end{array}$ & $\begin{array}{l}0.06 \\
(0.24)\end{array}$ & $\begin{array}{l}0.08 \\
(0.30)\end{array}$ \\
\hline Controls & Propensity score & All & Propensity score & All & Propensity score & All & Propensity score & All \\
\hline Minimum detectable effect & 17.14 & 21.08 & 27.66 & 34.2 & 0.67 & 0.86 & 0.67 & 0.85 \\
\hline \multirow[t]{2}{*}{$N$} & 145 & 145 & 145 & 145 & 145 & 145 & 145 & 145 \\
\hline & \multicolumn{2}{|c|}{$\begin{array}{l}\text { Oxycodone Equivalents } \\
(\mathrm{mg}) \text { per } 24 \mathrm{hr} \text { of } \\
\text { Hospitalization }\end{array}$} & \multicolumn{2}{|c|}{$\begin{array}{l}\text { Oxycodone Dose (mg) } \\
0-24 \text { hrs }\end{array}$} & \multicolumn{2}{|c|}{$\begin{array}{l}\text { Highest Daily Pain } \\
\text { Score }(0-10)\end{array}$} & \multicolumn{2}{|c|}{$\begin{array}{l}\text { Lowest Daily Pain } \\
\text { Score }(0-10)\end{array}$} \\
\hline General anesthesia & $\begin{array}{l}11.64 \\
(9.26)\end{array}$ & $\begin{array}{l}14.99 \\
(11.38)\end{array}$ & $\begin{array}{l}52.29 \\
(14.54)\end{array}$ & $\begin{array}{l}66.99 \\
(17.62)\end{array}$ & $\begin{array}{l}0.16 \\
(0.35)\end{array}$ & $\begin{array}{l}0.24 \\
(0.47)\end{array}$ & $\begin{array}{l}0.04 \\
(0.35)\end{array}$ & $\begin{array}{l}0.06 \\
(0.46)\end{array}$ \\
\hline Controls & Propensity score & All & Propensity score & All & Propensity score & All & Propensity score & All \\
\hline Minimum detectable effect & 26.12 & 32.09 & 41.02 & 49.70 & 0.99 & 1.31 & 0.99 & 1.31 \\
\hline $\mathrm{N}$ & 145 & 145 & 145 & 145 & 145 & 145 & 145 & 145 \\
\hline
\end{tabular}

Notes: Coefficients from ordinary least squares linear regressions. Top panel estimates the effect of methadone over buprenorphine. Bottom panel estimates effect of general anesthesia over neuraxial anesthesia. "Propensity score" calculated using all variables in Table I other than buprenorphine/methadone dosage and Medicaid status. "All" controls also refers to all variables in Table I less buprenorphine/methadone dosage and Medicaid status. "Minimum detectable effect" is smallest effect size expected to be found significant at $\alpha=0.05$ with $80 \%$ likelihood given standard error of main DV in regression. Standard errors in parentheses.

oxycodone $80 \mathrm{mg}$ is equivalent to oral morphine $120 \mathrm{mg} .{ }^{15}$ This is a similar range to previous studies in OUD patients, ${ }^{6,7}$ and much greater than other studies have shown most non-OUD patients take after cesarean delivery (oxycodone 17-33 mg per day). ${ }^{16,17}$ The high oxycodone requirements and high pain scores illustrate the historic difficulty of achieving effective post-cesarean analgesia for patients with OUD.

Anesthesia and adequate analgesia for these cesarean deliveries are complicated by key pharmacologic features of buprenorphine and methadone. Both medications have notably long half-lives (buprenorphine 24-37 hours, methadone 20-35 hours), and buprenorphine has a partial agonist ceiling effect as well as overwhelming mu opioid receptor affinity $\left(\mathrm{K}_{\mathrm{i}}\right.$ $0.22 \mathrm{nM}) .{ }^{11,18}$ As a result, few full agonist opioids can compete with buprenorphine. Sufentanil $\left(\mathrm{K}_{\mathrm{i}} 0.14\right)$ and hydromorphone $\left(\mathrm{K}_{\mathrm{i}}\right.$ 0.37) have affinities nearest to buprenorphine's, compared to fentanyl $\left(K_{i}\right.$ 1.35) and morphine $\left(\mathrm{K}_{\mathrm{i}} 1.17\right)$, but they are less commonly used or studied in obstetrical neuraxial anesthesia. ${ }^{18}$

Obstetric opinion has solidified that for pregnant women, even supervised withdrawal risks relapse, overdose, and catastrophic consequences. $^{2-5}$ Obstetricians maintain obstetric patients on buprenorphine or methadone through delivery, including cesarean section. The daily dose of buprenorphine or methadone is usually divided into 2-3 doses to account for increased volume of distribution and clearance. ${ }^{5,11}$ The optimal regimen for buprenorphine or methadone maintenance therapy at cesarean delivery, a major intraperitoneal surgery, remains under investigation. ${ }^{9,12-14}$

One small study of vaginal deliveries showed that women on either buprenorphine or methadone had good pain control and unremarkable opioid consumption, although the methadone group did require more NSAIDs. ${ }^{10}$ Larger cohort studies found that pain after vaginal delivery (but not opioid consumption) was higher in both buprenorphine and methadone patients compared to non-OUD controls. ${ }^{6,7}$ Cesarean cohorts in these studies had significantly greater pain scores and opioid consumption in both OUD treatment groups compared to controls. ${ }^{6,7}$ Another large cohort study clarified that opioid consumption after cesarean did not differ between buprenorphine and methadone patients. ${ }^{8}$ However, those studies did not provide anesthetic management details, such as medication dosing, that may have contributed to post-cesarean analgesic outcomes. 
The patients in our study were managed intraoperatively similarly to how non-OUD patients are managed at our institution. Our results agree with other studies that imply OUD patients likely need higher doses of neuraxial opioids. We expect opioid-tolerant patients to have fewer complaints of pruritis or nausea with a higher intrathecal opioid dose. Whether intrathecal hydromorphone, with its shorter duration but better competition with buprenorphine, is a better option than the standard of morphine also remains open for investigation. A recent metaanalysis compared non-OUD patients receiving spinal morphine $50-100 \mu \mathrm{g}$ to morphine $100-250 \mu \mathrm{g}$ for postcesarean analgesia. ${ }^{19}$ The higher morphine dose group had longer time to first analgesic request, although 24-hour opioid consumption was similar. The higher dose of spinal morphine also resulted in greater incidence of side-effects. Another study examined patients predicted to have severe pain after cesarean delivery, although not specifically patients with OUD. ${ }^{20}$ In that study, patients received either spinal morphine $300 \mu \mathrm{g}$ or $150 \mu \mathrm{g}$. The higher dose group had lower 24-hour pain scores, but 24-hour opioid consumption did not differ. No study comparing higher spinal morphine doses has been performed in obstetric patients with OUD maintained on buprenorphine or methadone.

Spinal clonidine use was infrequent among our patients. Meta-analyses show neuraxial clonidine prolongs motor and sensory blockade, reduces 24-hour opioid consumption, and prolongs time to first analgesic request after cesarean delivery compared to control patients, but whether these effects hold true in OUD patients has not been studied. ${ }^{21,22}$ Clonidine may be particularly beneficial in patients with OUD as it is a non-opioid analgesic. Clonidine's analgesia is multifactorial, it works in part via stimulation of post-synaptic $\alpha_{2}$-adrenergic receptors in the spinal cord at the dorsal horn and substantia gelatinosa, and by reducing afferent norepinephrine-mediated transmission of painful stimuli. ${ }^{21-23}$

Care of OUD parturients has several overlapping features with enhanced recovery after cesarean (ERAC). Both ERAC and OUD deliveries highlight a multi-modal, multitiered, and multi-disciplinary analgesic strategy. ${ }^{11,24,25}$ Previous case reports and small case series describe various postpartum analgesia strategies for OUD patients. These include IV opioid PCA, rescue IV dexmedetomidine or ketamine infusions, primary or rescue truncal blocks, and lumbar or thoracic continuous epidural infusions (local-only, local plus adjunct, and opioid-only). ${ }^{26-31}$ The key concerns of early ambulation, titratable analgesia, and unproven benefit (after intrathecal opioids or adjuncts in this special OUD population) limit what conclusions we can draw about each of these options. ${ }^{32,33}$ Nevertheless, the past several years have seen an increase in the ERAC and OUD discussions of multimodal strategies. This study provides an accurate example of how rarely these measures were done before the recent advocacy.

Another key component of multimodal therapy is scheduled acetaminophen and NSAIDs. ${ }^{11,34}$ In this study, patients received scheduled NSAID doses, although for the majority of the study period acetaminophen was only offered on patient request. Over the last few years of the study period, our institution began adhering to scheduled acetaminophen dosing. Over a similar period, but unrelatedly, methadone use became less frequent (Figure 4). The smaller acetaminophen amount in the methadone group is probably linked to these two distinct trends. The specific benefit of scheduled acetaminophen in OUD post-cesarean patients is predicted, but not proven. The link between less acetaminophen and more IV PCA use in our methadone group is provocative, suggesting more breakthrough coverage is needed if less scheduled, low-level analgesia is provided. Although not clearly proven, this supports scheduled acetaminophen.

Our study largely failed to show that buprenorphine or methadone dose could predict analgesic outcomes. Methadone dose did correlate weakly with average daily opioid requirement, but this connection was tenuous and not seen with pain scores. The daily buprenorphine dose did not correlate with pain scores or opioid consumption. One consideration involves the narrower range in buprenorphine doses, compared to the range in methadone doses. Perhaps larger sample sizes or a larger spread in dosing ranges would have teased apart a subtle, actual correlation. We also noted that our buprenorphine group was almost twice the size of the methadone group; this ratio reflects the growing preference for buprenorphine instead of methadone to treat OUD. Our study did not include a control (non-OUD) group or gather data on surgical duration or type of incision, which also relate to post-cesarean pain.

Neuraxial anesthesia for cesarean is preferred over general anesthesia, but this is for safety concerns. Closely related, but distinct, is the proven analgesic benefit of intrathecal morphine over truncal blocks and IV analgesia. ${ }^{32}$ Nevertheless, neither the literature nor our results permit a clear conclusion about which type of anesthetic results in better analgesia. In our study, although 
opioid requirement was reduced for the neuraxial group during the first 24 hours (the period we would expect to see a benefit from intrathecal morphine), early pain scores and time to first PACU analgesic were not different. The general anesthesia group in our study was commonly, but not universally, given long-acting opioid and the dose was not increased from the typical non-OUD dose. For now, safety more than clear analgesic benefit drives the preference for neuraxial anesthesia in OUD cesareans. ${ }^{11,24}$

In analyzing pain relief over time, the area under the curve (AUC) remains an ideal metric. Our results are limited by the fact that the medical records did not permit a useful AUC calculation. Instead, we substituted the blunter metric of the maximum daily pain score. In a prospective study, or with better records, an AUC should be used. The concern is that clinically significant, albeit statistically subtle differences in the analgesic effects may have gone unnoticed. Another concern is that pain scores (to calculate AUC) and breakthrough opioid use are clinically convenient but perhaps not sufficient markers of enhanced recovery. Our study did not include functional measures of analgesia or recovery. Like AUC, these outcomes would be more useful although less straightforward to collect without prospective, staff-intensive efforts, or changes in clinical practice.

\section{Conclusion}

This 8-year, single-center, retrospective study showed that cesarean delivery patients on buprenorphine or methadone receiving similar anesthetics had similar analgesia. Their pain scores and breakthrough opioid requirements were high compared to studies with non-OUD controls. Methadone dose only weakly correlated with postcesarean opioid requirement, and buprenorphine dose showed no correlation to pain scores or opioid requirement. This is the first report of analgesic outcomes in OUD parturients which includes anesthetic management details and may serve as a baseline for future investigations or in the clinical management of patients with OUD.

\section{Data Sharing Statement}

Requests for data should be addressed to the corresponding author.

\section{Ethics and Informed Consent}

Institutional review board (IRB) approval was obtained prior to beginning this investigation (IRB: 2014H0112). Given the retrospective nature of this study, no signed patient consent forms were required by the Ohio State University Institutional Review Board (IRB). Patient confidentiality was protected and this study was conducted in accordance with the Declaration of Helsinki.

\section{Acknowledgment}

The authors would like to thank Lucas C. Coffman, $\mathrm{PhD}$, for additional assistance with statistical analysis.

\section{Funding}

The authors report no sources of funding for this work.

\section{Disclosure}

Dr Mona R Prasad reports personal fees from Gilead, outside the submitted work. The authors report no other conflicts of interest associated with this work.

\section{References}

1. Maeda A, Bateman BT, Clancy CR, Creanga AA, Leffert LR. Opioid abuse and dependence during pregnancy: temporal trends and obstetrical outcomes. Anesthesiology. 2014;121(6):1158-1165. doi:10.10 97/ALN.0000000000000472

2. Committee on Obstetric Practice. Committee opinion no. 711: opioid use and opioid use disorder in pregnancy. Obstet Gynecol. 2017;130 (2):e81-e94. doi:10.1097/AOG.0000000000002235

3. Zedler BK, Mann AL, Kim MM, et al. Buprenorphine compared with methadone to treat pregnant women with opioid use disorder: a systematic review and meta-analysis of safety in the mother, fetus and child. Addiction. 2016;111(12):2115-2128. doi:10.1111/add.13 462

4. Meyer MC, Johnston AM, Crocker AM, Heil SH. Methadone and buprenorphine for opioid dependence during pregnancy: a retrospective cohort study. $J$ Addict Med. 2015;9(2):81-86. doi:10.1097/ADM.0000000000000092

5. Pan A, Zakowski M. Peripartum anesthetic management of the opioid-tolerant or buprenorphine/suboxone-dependent patient. Clin Obstet Gynecol. 2017;60:447-458.

6. Meyer M, Wagner K, Benvenuto A, Plante D, Howard D. Intrapartum and postpartum analgesia for women maintained on methadone during pregnancy. Obstet Gynecol. 2007;110(2, Part 1):261-266. doi:10.1097/01.AOG.0000275288.47258.e0

7. Meyer M, Paranya G, Keefer Norris A, Howard D. Intrapartum and postpartum analgesia for women maintained on buprenorphine during pregnancy. Eur J Pain. 2010;14(9):939-943. doi:10.1016/j.ejpain.20 10.03.002

8. Vilkins AL, Bagley SM, Hahn KA, et al. Comparison of post-cesarean section opioid analgesic requirements in women with opioid use disorder treated with methadone or buprenorphine. $J$ Addict Med. 2017;11(5):397-401. doi:10.1097/ADM.00000000000 00339

9. Wendling AL, Garvan C, Roussos-Ross D, Zhang L, Zeng D. Pain outcomes among patients after cesarean consuming buprenorphine or methadone and opioid-naïve patients. J Clin Anesth. 2020;65:109905. doi:10.1016/j.jclinane.2020.109905

10. Jones HE, O'Grady K, Dahne J, et al. Management of acute postpartum pain in patients maintained on methadone or buprenorphine during pregnancy. Am J Drug Alcohol Abuse. 2009;35(3):151-156. doi:10.1080/00952990902825413 
11. Landau R. Post-cesarean delivery pain: management of the opioid-dependent patient before, during and after cesarean delivery. Int J Obstet Anesth. 2019;39:105-116. doi:10.1016/j.ijoa.2019.01.011

12. Jonan AB, Kaye AD, Urman RD. Buprenorphine formulations: clinical best practice strategies recommendations for perioperative management of patients undergoing surgical or interventional pain procedures. Pain Physician. 2018;21:E1-E12.

13. Lembke A, Ottestad E, Schmiesing C. Patients maintained on buprenorphine for opioid use disorder should continue buprenorphine through the perioperative period. Pain Med. 2019;20(3):425-428. doi: $10.1093 / \mathrm{pm} / \mathrm{pny} 019$

14. Anderson TA, Quaye AN, Ward EN, et al. To stop or not, that is the question: acute pain management for the patient on chronic buprenorphine. Anesthesiology. 2017;126(6):1180-1186. doi:10.10 97/ALN.0000000000001633

15. McPherson ML. Demystifying Opioids Conversion Calculations: A Guide for Effective Dosing. ASHP: Bethesda; 2009.

16. Prabhu M, Dolisca S, Wood R, et al. Postoperative opioid consumption after scheduled compared with unscheduled cesarean delivery. Obstet Gynecol. 2019;133(2):354-363. doi:10.1097/AOG.00000000 00003087

17. Lester SA, Kim B, Tubinis MD, Morgan CJ, Powell MF. Impact of an enhanced recovery program for cesarean delivery on postoperative opioid use. Int J Obstet Anesth. 2020;43:47-55. doi:10.1016/j.ijoa.2020.01.005

18. Volpe DA, McMahon Tobin GA, Mellon RD, et al. Uniform assessment and ranking of opioid $\mathrm{Mu}$ receptor binding constants for selected opioid drugs. Regul Toxicol Pharmacol. 2011;59 (3):385-390. doi:10.1016/j.yrtph.2010.12.007

19. Sultan P, Halpern SH, Pushpanathan E, Patel S, Carvalho B. The effect of intrathecal morphine dose on outcomes after elective cesarean delivery: a meta-analysis. Anesth Analg. 2016;123(1):154-164. doi:10.1213/ANE.0000000000001255

20. Booth JL, Harris LC, Eisenach JC, Pan PH. A randomized controlled trial comparing two multimodal analgesic techniques in patients predicted to have severe pain after cesarean delivery. Anesth Analg. 2016;122(4):1114-1119. doi:10.1213/ANE.0000000000000695

21. Crespo S, Dangelser G, Haller G. Intrathecal clonidine as an adjuvant for neuraxial anaesthesia during caesarean delivery: a systematic review and meta-analysis of randomised trials. Int J Obstet Anesth. 2017;32:64-76. doi:10.1016/j.ijoa.2017.06.009

22. Allen TK, Mishriky BM, Klinger RY, Habib AS. The impact of neuraxial clonidine on postoperative analgesia and perioperative adverse effects in women having elective caesarean section-a systematic review and meta-analysis. $\mathrm{Br} J$ Anaesth. 2018;120(2):228-240. doi:10.1016/j.bja.2017.11.085

23. Filos KS, Goudas LC, Patroni O, Polyzou V. Intrathecal clonidine as a sole analgesic for pain relief after cesarean section. Anesthesiology. 1992;77(2):267-274. doi:10.1097/00000542-199208000-00008
24. California Maternal Quality Care Collaborative [homepage on the Internet]. Mother \& baby substance exposure toolkit best practices: labor and delivery; 2020. Available from: https://nastoolkit.org/audi ence/labor-delivery. Accessed August 19, 2020.

25. Krans EE, Campopiano M, Cleveland LM, et al. National partnership for maternal safety consensus bundle on obstetric care for women with opioid use disorder. Obstet Gynecol. 2019;134(2):365-375. doi:10.1097/AOG.0000000000003381

26. Hoyt MR, Shah U, Cooley J, Temple M. Use of epidural clonidine for the management of analgesia in the opioid addicted parturient on buprenorphine maintenance therapy: an observational study. Int J Obstet Anesth. 2018;34:67-72. doi:10.1016/j.ijoa.20 18.01.001

27. Leighton BL, Crock LW. Case series of successful postoperative pain management in buprenorphine maintenance therapy patients. Anesth Analg. 2017;125(5):1779-1783. doi:10.1213/ANE.000000000 0002498

28. Tith S, Bining G, Bollag L. Management of eight labor and delivery patients dependent on buprenorphine (Subutex ${ }^{\mathrm{TM}}$ ): a retrospective chart review. F1000Res. 2018;7:7. doi:10.12688/f1000research. 13350.1

29. Jones HE, Johnson RE, Milio L. Post-cesarean pain management of patients maintained on methadone or buprenorphine. Am J Addict. 2006;15(3):258-259. doi:10.1080/10550490600626721

30. Wasiluk IM, Castillo D, Panni JK, Stewart S, Panni MK. Postpartum analgesia with dexmedetomidine in opioid tolerance during pregnancy. J Clin Anesth. 2011;23(7):593-594. doi:10.1016/j. jclinane.2010.09.013

31. Coffman JC, Fiorini K, Small RH. Ilioinguinal-iliohypogastric block used to rescue ineffective transversus abdominis plane block after cesarean delivery. Int $J$ Obstet Anesth. 2015;24(4):394-395. doi:10.1016/j.ijoa.2015.05.003

32. Patel SD, Sharawi N, Sultan P. Local anaesthetic techniques for post-caesarean delivery analgesia. Int J Obstet Anesth. 2019; 40:62-77. doi:10.1016/j.ijoa.2019.06.002

33. Krohg A, Ullensvang K, Rosseland LA, Langesaeter E, Sauter AR. The analgesic effect of ultrasound-guided quadratus lumborum block after cesarean delivery: a randomized clinical trial. Anesth Analg. 2018;126(2):559-565. doi:10.1213/ANE.0000000000 002648

34. Valentine AR, Carvalho B, Lazo TA, Riley ET. Scheduled acetaminophen with as-needed opioids compared to as-needed acetaminophen plus opioids for post-cesarean pain management. Int J Obstet Anesth. 2015;24(3):210-216. doi:10.1016/j.ijoa.20 15.03.006
Journal of Pain Research

\section{Publish your work in this journal}

The Journal of Pain Research is an international, peer reviewed, open access, online journal that welcomes laboratory and clinical findings in the fields of pain research and the prevention and management of pain. Original research, reviews, symposium reports, hypothesis formation and commentaries are all considered for publication. The manuscript management system is completely online and includes a very quick and fair peer-review system, which is all easy to use. Visit http:// www.dovepress.com/testimonials.php to read real quotes from published authors. 